\title{
The Ernst equation and ergosurfaces
}

\author{
Piotr T Chruściel ${ }^{1,5}$, Gert-Martin Greuel ${ }^{2}$, Reinhard Meinel ${ }^{3}$ \\ and Sebastian J Szybka ${ }^{4}$ \\ ${ }^{1}$ LMPT, Fédération Denis Poisson, Tours, France \\ 2 Universität Kaiserslautern, Kaiserslautern, Germany \\ ${ }^{3}$ Universität Jena, Jena, Germany \\ ${ }^{4}$ Obserwatorium Astronomiczne UJ, Kraków, Poland \\ E-mail: Piotr.Chrusciel@lmpt.univ-tours.fr, greuel@mathematik.uni-kl.de, \\ meinel@tpi.uni-jena.de and szybka@if.uj.edu.pl
}

Received 6 March 2006, in final form 9 May 2006

Published 9 June 2006

Online at stacks.iop.org/CQG/23/4399

\begin{abstract}
We show that analytic solutions $\mathscr{E}$ of the Ernst equation with a non-empty zero-level set of $\Re \mathscr{E}$ lead to smooth ergosurfaces in spacetime. In fact, the spacetime metric is smooth near an 'Ernst ergosurface' $E_{f}$ if, and only if, $\mathscr{E}$ is smooth near $E_{f}$ and does not have zeros of infinite order there.
\end{abstract}

PACS numbers: 04.20.CV, 04.20.Dw

(Some figures in this article are in colour only in the electronic version)

\section{Introduction}

A standard procedure for constructing stationary axi-symmetric solutions of the Einstein equations is a reduction of the Einstein equations to a two-dimensional nonlinear equationthe Ernst equation [5] — using the asymptotically timelike Killing vector field $X$ as the starting point of the reduction: one finds a complex-valued field

$$
\mathscr{E}=f+\mathrm{i} b,
$$

by, e.g. solving a boundary-value problem [17]. The spacetime metric is then obtained by solving ODEs for the metric functions. The following difficulties arise in this construction:

(i) Singularities of $\mathscr{E}$, which might—or might not—lead to singularities of the metric.

(ii) Struts or causality violations at the rotation axis.

(iii) Singularities of the equations arising at zeros of $\Re \mathscr{E}$.

5 Current address: A Einstein Institute, Golm, Germany. 
The aim of this work is to address this last question. Indeed, the equations determining the metric functions are singular at the zero-level set ${ }^{6}$

$$
E_{f}:=\{f=0, \rho>0\}
$$

of $f:=\Re \mathscr{E}$; we will refer to $E_{f}$ as the $\mathscr{E}$-ergosurface. We show, assuming smoothness of $\mathscr{E}$ in a neighbourhood of $E_{f}$, and excluding zeros of infinite order, that the singularities of the solutions of those ODEs conspire to produce a smooth spacetime metric. More precisely, we have

Theorem 1.1. Consider a smooth solution $f+\mathrm{i} b$ of the Ernst equations (2.5)-(2.6) below such that $f$ has no zeros of infinite order at the $\mathscr{E}$-ergosurface $E_{f}$. Then there exists a neighbourhood of $E_{f}$ on which the metric (2.1) obtained by solving (2.3)-(2.4) is smooth and has a Lorentzian signature.

An immediate corollary of theorem 1.1 is the following: any point $\vec{x}_{0}$ off the axis in the Weyl coordinate chart corresponds to spacetime points at which the metric is regular $\Longleftrightarrow$ the Ernst potential is a real-analytic function of the Weyl coordinates near $\vec{x}_{0} \Longleftrightarrow$ the Ernst potential is a smooth function of the Weyl coordinates near $\vec{x}_{0}$ and zeros of $\mathfrak{R} \mathscr{E}$ have finite order.

The proof of theorem 1.1 can be found at the end of section 5.2.

The condition of zeros of finite order is necessary, in the following sense: any zero of $\Re \mathscr{E}$ on a smooth spacetime ergosurface is of finite order. This is proved at the end of section 2 .

It is an interesting consequence of our analysis below that a critical zero of $f$ of order $k$ corresponds to a smooth two-dimensional surface in spacetime at which $k$ distinct components of the ergoregion $\{f<0\}$ 'almost meet', in the sense that their closures intersect there, with the boundaries branching out. Two exact solutions with this behaviour for $k=2$ are presented in section 5 .

Section 4 below appeared in preprint form in [4]; the reader will also find in [4] some more information about second order zeros of $f$.

\section{The field equations and ergosurfaces}

We consider a vacuum gravitational field in Weyl-Lewis-Papapetrou coordinates

$$
\mathrm{d} s^{2}=f^{-1}\left[h\left(\mathrm{~d} \rho^{2}+\mathrm{d} \zeta^{2}\right)+\rho^{2} \mathrm{~d} \phi^{2}\right]-f(\mathrm{~d} t+a \mathrm{~d} \phi)^{2}
$$

with all functions depending only upon $\rho$ and $\zeta$. The vacuum Einstein equations for the metric functions $h, f$ and $a$ are equivalent to the Ernst equation

$$
(\Re \mathscr{E})\left(\mathscr{E}_{, \rho \rho}+\mathscr{E}_{, \zeta \zeta}+\frac{1}{\rho} \mathscr{E}_{, \rho}\right)=\mathscr{E}_{, \rho}^{2}+\mathscr{E}_{, \zeta}^{2}
$$

for the complex function $\mathscr{E}(\rho, \zeta)=f(\rho, \zeta)+\mathrm{i} b(\rho, \zeta)$, where $b$ replaces $a$ via

$$
a_{, \rho}=\rho f^{-2} b_{, \zeta}, \quad a_{, \zeta}=-\rho f^{-2} b_{, \rho}
$$

and $h$ can be calculated from

$$
h_{, \rho}=\frac{\rho h}{2 f^{2}}\left[f_{, \rho}^{2}-f_{, \zeta}^{2}+b_{, \rho}^{2}-b_{, \zeta}^{2}\right], \quad h_{, \zeta}=\frac{\rho h}{f^{2}}\left[f_{, \rho} f_{, \zeta}+b_{, \rho} b_{, \zeta}\right] .
$$

6 The equations are of course singular at $f=\rho=0$ as well, but the singularity of the whole system of equations has a different nature there, because of the $\partial_{\rho} f / \rho$ terms in the Ernst equation (2.2), and will not be considered here. Geometrically, the set $\{\rho=f=0\}$ has a rather different nature, corresponding to Killing horizons, with the boundary conditions there being reasonably well understood in any case $[3,10]$. 
We will think of $\rho$ and $\zeta$ as being cylindrical coordinates in $\mathbb{R}^{3}$ equipped with the flat metric

$$
\stackrel{\circ}{g}=\mathrm{d} \rho^{2}+\rho^{2} \mathrm{~d} \varphi^{2}+\mathrm{d} \zeta^{2},
$$

with all the above functions being $\varphi$-independent functions on $\mathbb{R}^{3}$. Then (2.2) can be rewritten as

$$
\begin{aligned}
& f \Delta f=|D f|^{2}-|D b|^{2}, \\
& f \Delta b=2(D f, D b),
\end{aligned}
$$

where $\Delta$ is the flat Laplace operator of the metric $\stackrel{\circ}{g}$, and $(\cdot, \cdot)$ denotes the $\stackrel{\circ}{g}$-scalar product, similarly the norm $|\cdot|$ is the one associated with $g$.

Equations (2.5)-(2.6) degenerate at $\{f=0\}$, and it is not clear that $f$ or $b$ will smoothly extend across $\{f=0\}$, if at all. In section 3 we give examples of solutions which do not. On the other hand, there are large classes of solutions which are smooth across $\mathscr{E}_{f}$. Examples can be obtained as follows:

First, every spacetime obtained from an Ernst map $\mathscr{E} \prime$ associated with the reduction that uses the axial Killing vector $\partial_{\varphi}$ (see, e.g., $[3,21]$ ) will lead to a solution $\mathscr{E}$ as considered here that extends smoothly across the spacetime ergosurfaces (if any), recall that an ergosurface is defined to be a timelike hypersurface where the Killing vector $X$, which asymptotes a time translation in the asymptotic region, becomes null. Those ergosurfaces correspond then to $\mathscr{E}$-ergosurfaces across which $f$ does indeed extend smoothly. We emphasize that we are interested in the construction of a spacetime starting from $\mathscr{E}$, and we have no a priori reason to expect that an $\mathscr{E}$-ergosurface, defined as smooth zero-level set of $\Re \mathscr{E}$, will lead to a smooth spacetime ergosurface.

Next, large classes of further examples are given in $[9,12,14-17,22]^{7}$. Some of the solutions in those references have non-trivial zero-level sets of $\Re \mathscr{E}$, with $g_{\rho \rho}=g_{z z}$ and $g_{t \varphi}$ smooth across $E_{f}$ (see in particular [12]), but smoothness of $g_{\varphi \varphi}$ is not manifest.

Quite generally, we have the following: consider a vacuum spacetime $(\mathscr{M}, g)$ with two Killing vectors $X, Y$, and with a non-empty spacetime ergosurface defined as

$$
E_{\mathscr{M}}:=\{\underbrace{g(X, X)=0, X \neq 0}_{(1)}, \underbrace{g(X, X) g(Y, Y)-g(X, Y)^{2}<0}_{(2)}\} .
$$

Condition (1) is the statement that $X$ becomes null on $E_{\mathscr{M}}$, while (2) says that the planes spanned by $X$ and $Y$ are timelike; condition (2) distinguishes a spacetime ergosurface from a horizon, where those planes are null. (For solutions in Weyl form, condition (2) translates into the requirement $\rho \neq 0$.) Now, by (2) there exists a linear combination of $X$ and $Y$ which is timelike near $E_{\mathscr{M}}$, and if $g$ is sufficiently differentiable $\left(H_{\text {loc }}^{2}\right.$ in coordinates adapted to the symmetry group is more than enough), the analysis of [13] shows that there exists an atlas near $E_{\mathscr{M}}$ in which $g$ is analytic. By chasing through the construction of Weyl coordinates, this implies that $f$ and $b$ are real-analytic functions near $E_{f}$. In particular $f$ will not have zeros of infinite order there.

\section{Static solutions}

In the remainder of this work only those solutions which are invariant under rotations around some fixed chosen axis are considered (when viewed as functions on subsets of $\mathbb{R}^{3}$ ), and all functions are identified with functions of two variables, $\rho$ and $\zeta$.

\footnotetext{
7 The solutions we are referring to here are not necessarily vacuum everywhere, and some of them have a function $\mathscr{E}$ which is singular somewhere in the $(\rho, \zeta)$ plane. Our analysis applies to the vacuum region, away from the rotation axis, and away from the singularities of the Ernst map $f+\mathrm{i} b$.
} 
Consider a solution of (2.5)-(2.6) with $b \equiv 0$. Setting $u=\ln f$ in the region $\Omega:=$ $\{f>0\}$, equation (2.5) becomes

$$
\Delta u=0 \quad \text { on } \Omega \text {. }
$$

One can now obtain examples of solutions for which $E_{f}$ is not empty as follows: let $\alpha \in \mathbb{R}^{*}, \vec{x}_{0}=\left(\rho_{0}, \zeta_{0}\right) \in(0, \infty) \times \mathbb{R}$; standard PDE considerations show existence of solutions of $(3.1)$ on $\Omega:=\{(0, \infty) \times \mathbb{R}\} \backslash\left\{\vec{x}_{0}\right\}$ such that

$$
u_{\alpha}=\alpha \ln \left(\left(\rho-\rho_{0}\right)^{2}+\left(\zeta-\zeta_{0}\right)^{2}\right)+O(1) \text {. }
$$

This leads to

$$
f_{\alpha}:=e^{u_{\alpha}}=\left(\left(\rho-\rho_{0}\right)^{2}+\left(\zeta-\zeta_{0}\right)^{2}\right)^{\alpha} g_{\alpha},
$$

where $g_{\alpha}$ has no zeros. We have the following:

- No such solution is smoothly extendable through the Ernst ergosurface $E_{f_{\alpha}}=\left\{\vec{x}_{0}\right\}$ except perhaps when $\alpha \in \mathbb{N}^{*}$.

- In that last case the solutions do not extend smoothly across $E_{f}$ either, which can be seen as follows. Consider first $\alpha=1$, then $f=f_{1}$ has a zero of order 2 with positive-definite Hessian, but lemma 5.2 shows that no such solutions which are smooth across $E_{f}$ exist. For general $\alpha=n \in \mathbb{N}^{*}$ we note that

$$
f_{1}=\left(\left(\rho-\rho_{0}\right)^{2}+\left(\zeta-\zeta_{0}\right)^{2}\right)\left(g_{n}\right)^{\frac{1}{n}} .
$$

But smoothness of $f_{n}$ would imply that of $g_{n}$, and thus of $f_{1}$, which is not the case. Thus $f_{n}, n \in \mathbb{N}^{*}$, cannot be smooth either.

Above we have considered differentiability of $f_{\alpha}$ in $(\rho, \zeta)$-coordinates. This might not be equivalent to the question which is of main interest here, that of the regularity of the spacetime metric. In the case $b \equiv 0$ this issue is easy to handle, by noting that $a$ can then always be made to vanish by a redefinition of $t$. Now, the length $g\left(\partial_{\varphi}, \partial_{\varphi}\right)$ of the Killing vector $\partial_{\varphi}$, generating rotations around the axis, is a smooth-hence locally bounded-function on the spacetime. But $g_{\varphi \varphi}=\rho^{2} f^{-1}$ by (2.1) so, in the static case, zeros of $f$ with $\rho_{0} \neq 0$ cannot correspond to ergosurfaces in spacetime ${ }^{8}$.

\section{Non-critical zeros of $f$}

We start with the following:

Theorem 4.1. The conclusion of theorem 1.1 holds if one moreover assumes that $|D f|$ has no zeros at the $\mathscr{E}$-ergosurface $E_{f}:=\{f=0, \rho>0\}$.

Proof. We need to show that the functions

$$
\alpha:=-g_{\varphi t}=a f, \quad \beta:=\ln g_{\zeta \zeta}=\ln g_{\rho \rho}=\ln \left(h f^{-1}\right),
$$

as well as

$$
g_{\varphi \varphi}=\frac{\rho^{2}-(a f)^{2}}{f}
$$

are smooth across $\{f=0, \rho>0\}$, and that $g_{\varphi t}$ does not vanish whenever $g_{t t}=-f$ does.

8 The discussion here gives a simple proof, under the supplementary condition of axi-symmetry, of the VishweshwaraCarter lemma, that there are no ergosurfaces in static spacetimes. 
We start by Taylor expanding $f$ and $b$ to order 2 near any point $\left(\rho_{0}, \zeta_{0}\right)$ such that $f\left(\rho_{0}, \zeta_{0}\right)=0$ :

$$
\begin{aligned}
& f(\rho, \zeta)=\dot{f}_{, \rho}\left(\rho-\rho_{0}\right)+\dot{\circ}_{, \zeta}\left(\zeta-\zeta_{0}\right) \\
& +\frac{1}{2} \stackrel{\circ}{f, \rho \rho}\left(\rho-\rho_{0}\right)^{2}+\frac{1}{2} \dot{f}_{, \zeta \zeta}\left(\zeta-\zeta_{0}\right)^{2}+\stackrel{\circ}{f}, \rho \zeta_{,}\left(\rho-\rho_{0}\right)\left(\zeta-\zeta_{0}\right)+\cdots,
\end{aligned}
$$

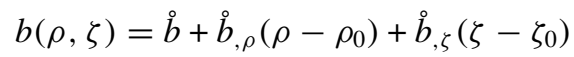

$$
\begin{aligned}
& +\frac{1}{2} \stackrel{\circ}{b}, \rho \rho_{0}\left(\rho-\rho_{0}\right)^{2}+\frac{1}{2} \stackrel{\circ}{, \zeta \zeta}_{\zeta}\left(\zeta-\zeta_{0}\right)^{2}+\stackrel{\circ}{b}, \rho \zeta\left(\rho-\rho_{0}\right)\left(\zeta-\zeta_{0}\right)+\cdots,
\end{aligned}
$$

where a circle over a function indicates that the value at $\rho_{0}$ and $\zeta_{0}$ is taken. Inserting these expansions into (2.5)-(2.6), after tedious but elementary algebra one obtains either

$\dot{b}_{\rho}=\mp \dot{f}_{\zeta}$,

$$
\grave{b}_{\zeta}= \pm \dot{f}_{\rho} \text {, }
$$

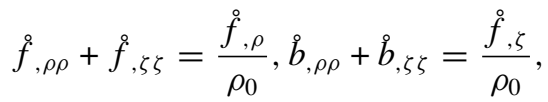

$$
\stackrel{\circ}{b}, \rho \zeta=\stackrel{\circ}{f}_{, \zeta \zeta}, \quad \stackrel{\circ}{f, \rho \zeta}=\stackrel{\circ}{b, \rho \rho},_{,}
$$

or

$$
\grave{b}_{\rho}=\dot{f}_{\zeta}=\grave{b}_{\zeta}=\dot{f}_{\rho}=0 .
$$

The second possibility is excluded by our hypothesis that $D f \neq 0$ on $E_{f}$.

Suppose, first, that the lower signs arise in the first line of (4.1). From (2.3) we obtain

$$
\begin{aligned}
& \alpha_{, \rho}=\frac{f_{, \rho}}{f} \alpha+\frac{\rho}{f} b_{, \zeta}, \\
& \alpha_{, \zeta}=\frac{f_{, \zeta}}{f} \alpha-\frac{\rho}{f} b_{, \rho},
\end{aligned}
$$

so that

$$
\begin{aligned}
& \left(\frac{\alpha-\rho}{f}\right)_{, \rho}=\underbrace{\left[\rho\left(b_{, \zeta}+f_{, \rho}\right)-f\right]}_{=: \sigma_{\rho}} f^{-2}, \\
& \left(\frac{\alpha-\rho}{f}\right)_{, \zeta}=\underbrace{\rho\left(f_{, \zeta}-b_{, \rho}\right)}_{=: \sigma_{\zeta}} f^{-2} .
\end{aligned}
$$

Inserting (4.1) into the definitions of $\sigma_{\rho}$ and $\sigma_{\zeta}$ we find

$$
\sigma_{\rho}=\sigma_{\zeta}=0=\mathrm{d} \sigma_{\rho}=\mathrm{d} \sigma_{\zeta}
$$

at every point $\left(\rho_{0}, \zeta_{0}\right)$ lying on the $\mathscr{E}$-ergosurface. Here, as elsewhere, $\mathrm{d} \mu$ denotes the differential of a function $\mu$.

Recall that $D f$ does not vanish on $E_{f}$. We can thus introduce coordinates $(x, y)$ near each connected component of $E_{f}$ so that $f=x$. Since the $\sigma_{a}$ are smooth we have the Taylor expansions

$$
\sigma_{a}=\left.\sigma_{a}\right|_{E_{f}}+\left.\left(\partial_{x} \sigma_{a}\right)\right|_{E_{f}} x+r_{a} x^{2},
$$

for some remainder terms $r_{a}$ which are smooth functions on spacetime. But we have shown that $\left.\sigma_{a}\right|_{E_{f}}=\left.\left(\partial_{x} \sigma_{a}\right)\right|_{E_{f}}=0$. Hence

$$
\sigma_{a}=r_{a} x^{2}=r_{a} f^{2} .
$$

It follows that the right-hand sides of (4.5)-(4.6) extend by continuity across $E_{f}$ to smooth functions. Hence the derivatives of $(\alpha-\rho) / f$ extend by continuity to smooth functions, and by integration

$$
\alpha-\rho=f \hat{\alpha},
$$




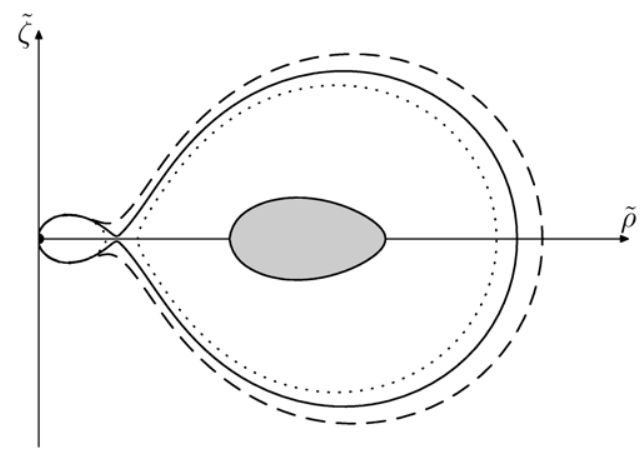

Figure 1. A coordinate representation of ergosurfaces for three configurations consisting of a black hole (coordinate origin) surrounded by a fluid ring (shaded area). The values of the parameters, in the notation of [2], are $\varrho_{\mathrm{i}} / \varrho_{\mathrm{o}}=0.55, r_{\mathrm{c}} / \varrho_{\mathrm{o}}=0.015, J_{\mathrm{c}} / \varrho_{\mathrm{o}}^{2}=0.05$, with $V_{0}=-1.45$ (dashed line), $V_{0}=-1.396$ (solid line) and $V_{0}=-1.35$ (dotted line). The shape of the ring (here corresponding to the ergosurface indicated by a solid line) is represented by the shaded area and differs only minimally between the three configurations. The coordinates $\varrho$ and $\tilde{\zeta}$ are related to $\varrho$ and $\zeta$ by a conformal transformation.

for some smooth function $\hat{\alpha}(\rho, \zeta)$. This proves smoothness of both $g_{t \varphi}$ and $g_{\varphi \varphi}$. We also obtain that $g_{t \varphi}=-\rho$ when $f=0$, and since $\rho>0$ by assumption we obtain non-vanishing of $g_{t \varphi}$ on the $\mathscr{E}$-ergosurface.

In the case where the upper choice of sign in (4.1) occurs, instead of (4.5)-(4.6) we write equations for $(\alpha+\rho) / f$, and an identical argument applies.

We pass now to the analysis of $g_{\rho \rho}=g_{z z}$. From (2.4),

$$
\begin{aligned}
& \ln (h / f)_{, \rho}=\underbrace{\frac{1}{2}\left[\rho\left(f_{, \rho}^{2}-f_{, \zeta}^{2}+b_{, \rho}^{2}-b_{, \zeta}^{2}\right)-2 f f_{, \rho}\right]}_{=: \kappa_{\rho}} f^{-2}, \\
& \ln (h / f)_{, \zeta}=\underbrace{\left[\rho\left(f_{, \rho} f_{, \zeta}+b_{, \rho} b_{, \zeta}\right)-f f_{, \zeta}\right]}_{=: \kappa_{\zeta}} f^{-2} .
\end{aligned}
$$

Evaluating $\kappa_{a}$ and its derivatives on $E_{f}$ and using (4.1) one obtains again

$$
\kappa_{a}=\mathrm{d} \kappa_{a}=0
$$

on $E_{f}$. As before we conclude that $g_{\rho \rho}$ and $g_{\zeta \zeta}$ are smooth across $E_{f}$.

\section{Higher order zeros of $f$}

We shall say that $f$ has a zero of order $n, n \geqslant 1$, at $\vec{x}_{0}=\left(\rho_{0}, \zeta_{0}\right)$, if

$$
f\left(\vec{x}_{0}\right)=\cdots=\underbrace{D \cdots D}_{n-1 \text { factors }} f\left(\vec{x}_{0}\right)=0 \text { but } \underbrace{D \cdots D}_{n \text { factors }} f\left(\vec{x}_{0}\right) \neq 0 .
$$

It is legitimate to raise the question of whether solutions of the Ernst equations (2.2) with higher order zeros on $E_{f}$ exist. A simple mechanism ${ }^{9}$ for producing such solutions is the following: consider a family of solutions depending continuously on one parameter, such that for large parameter values there exist two disjoint ergosurfaces, while for small parameter

\footnotetext{
9 We are grateful to M Ansorg and D Petroff for pointing this out to us.
} 

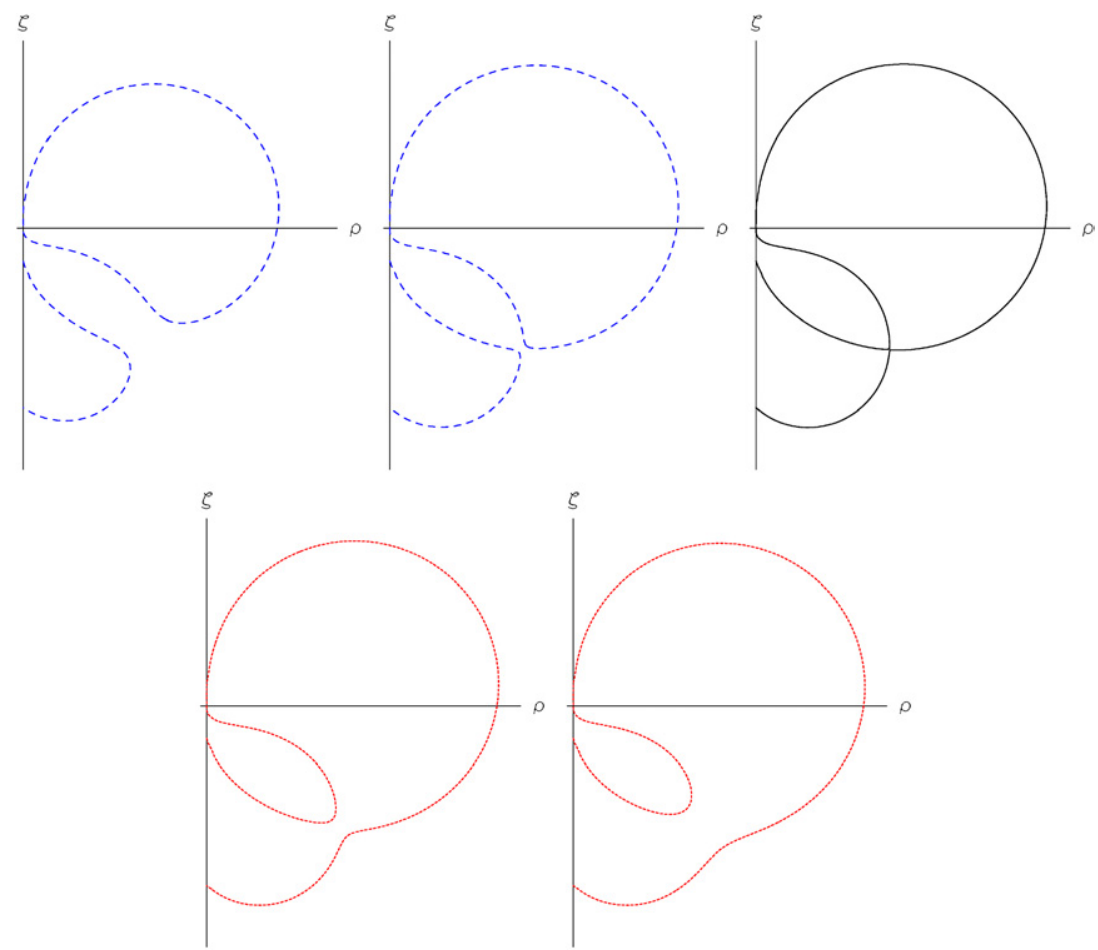

Figure 2. Coalescing ergosurfaces in the 'double-Kerr' family of metrics in the $(\varrho, \zeta)$ half-plane, with one black hole extreme. We use a parametrization as in [20]. In all five cases the event horizon of the degenerate black hole lies at the origin, thus $\alpha_{1}=0$, while $\alpha_{2}=-1 / 6, \alpha_{3}=-1$, and with the $\beta_{a}, a=1,2$ of the form $\beta_{a}=-b_{a}(1+\mathrm{i})$, where (1) $b_{1}=0.6, b_{2}=1.5$; (2) $b_{1}=0.62, b_{2}=1.66$; (3) $b_{1}=0.6218704381, b_{2}=1.668809562$; (4) $b_{1}=0.62, b_{2}=$ 1.68 ; (5) $b_{1}=0.6, b_{2}=1.7$. Those solutions have both singular struts at the axis and singular rings away from the region where the coalescing of ergosurfaces occurs, but those singularities are irrelevant for the proof that there are no local obstructions to zeros of higher order.

values the ergosurface is connected. Elementary considerations show that there exists a value of the parameter where $f$ has a zero of higher order. Examples of such behaviour have been found numerically by Ansorg [1] in families of differentially rotating discs (however, the merging of the ergosurfaces in that work occurs in the matter region, which is not covered by our analysis). In figure 1 , due to D Petroff ${ }^{10}$, the reader will find an example in a family of solutions with a black hole surrounded by a ring of fluid. Those solutions are globally regular, and the coalescence of ergosurfaces takes place in the vacuum region. A purely vacuum example of this kind is hinted at in [18, figure 2]. Finally, figures 2-4 show a purely vacuum example within the Kramer-Neugebauer family of solutions [9], where the parameters which are being varied are the $\beta_{i}$ of [20]. While the value of the parameters found numerically, for which $f$ has a zero of order 2 , is only approximate, the existence of a nearby value with a second order zero follows from what has been said above together with the remaining results in this paper.

Similarly three ergosurfaces merging simultaneously will lead to a zero of order precisely 3 , and so on.

${ }^{10}$ We are very grateful to D Petroff for providing this figure; a detailed analysis of configurations of this type can be found in [2]. 


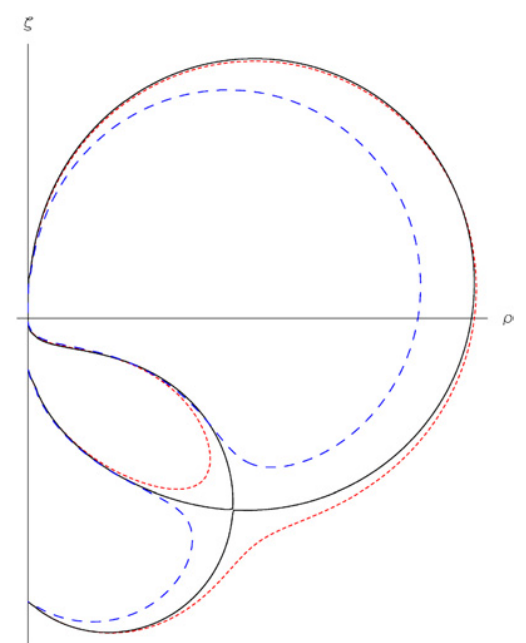

Figure 3. The first, third and fifth ergosurfaces of figure 2 superimposed.

In order to study the zeros of higher order it is convenient to consider Taylor expansions of $f$ and $b$ to order $n \geqslant k$,

$$
f(\rho, \zeta)=\sum_{0 \leqslant i+j \leqslant n} \frac{\dot{f}_{i, j}}{i ! j !}\left(\rho-\rho_{0}\right)^{i}\left(\zeta-\zeta_{0}\right)^{j}+r_{n},
$$

where

$$
\stackrel{\circ}{f}_{i, j}:=\frac{\partial^{i+j} f}{\partial^{i} \rho \partial^{j} \zeta}\left(\rho_{0}, \zeta_{0}\right) .
$$

Similarly we denote the Taylor coefficients of $b$ by $\stackrel{\circ}{i, j}_{j}$.

Suppose that $f$ has a zero of order $k$ at $\vec{x}_{0}$. Note that the value $b\left(\rho_{0}, \zeta_{0}\right)=\mathfrak{s} \mathscr{E}\left(\rho_{0}, \zeta_{0}\right)$ is irrelevant both for the equations and for the metric, so without loss of generality we may assume that $\mathscr{E}\left(\rho_{0}, \zeta_{0}\right)=0$. From now on we assume that this is the case. Let $\mathscr{E}_{k}$ be the homogeneous polynomial in $\rho-\rho_{0}$ and $\zeta-\zeta_{0}$, of order $k$, obtained by keeping in the Taylor expansion only the terms of first non-vanishing order, similarly $f_{k}$. Thus, $f_{k}$ is a homogeneous polynomial in $\rho-\rho_{0}$ and $\zeta-\zeta_{0}$, of order $k$ :

$$
f_{k}(\rho, \zeta)=\sum_{i+j=k} \frac{\stackrel{\circ}{f}_{i, j}}{i ! j !}\left(\rho-\rho_{0}\right)^{i}\left(\zeta-\zeta_{0}\right)^{j} .
$$

The polynomial $f_{k}$ can be written in a convenient form, (5.4) below, as follows: suppose, for the moment, that $\rho-\rho_{0}$ is strictly positive, by homogeneity we can then write

$$
f_{k}(\rho, \zeta)=\left(\rho-\rho_{0}\right)^{k} P_{k}(w), \quad \text { where } \quad w:=\frac{\zeta-\zeta_{0}}{\rho-\rho_{0}}
$$

for some non-trivial polynomial $P_{k}$ of order smaller than or equal to $k$. Let $\alpha_{i} \in \mathbb{C}, i=$ $1, \ldots, n$, be distinct zeros of $P_{k}$, with multiplicities $m_{i}$, thus $P_{k}(w)=C \Pi_{i=1}^{n}\left(w-\alpha_{i}\right)^{m_{i}}$, for some constant $C \in \mathbb{C}^{*}$. Hence

$$
f_{k}(\rho, \zeta)=C\left(\rho-\rho_{0}\right)^{m_{0}} \Pi_{i=1}^{n}\left(\zeta-\zeta_{0}-\alpha_{i}\left(\rho-\rho_{0}\right)\right)^{m_{i}},
$$

where $m_{0}=k-m_{1}-\cdots m_{n}$. It should be clear that (5.4) remains true for all $\rho$, and not only for $\rho>\rho_{0}$ as assumed so far. 

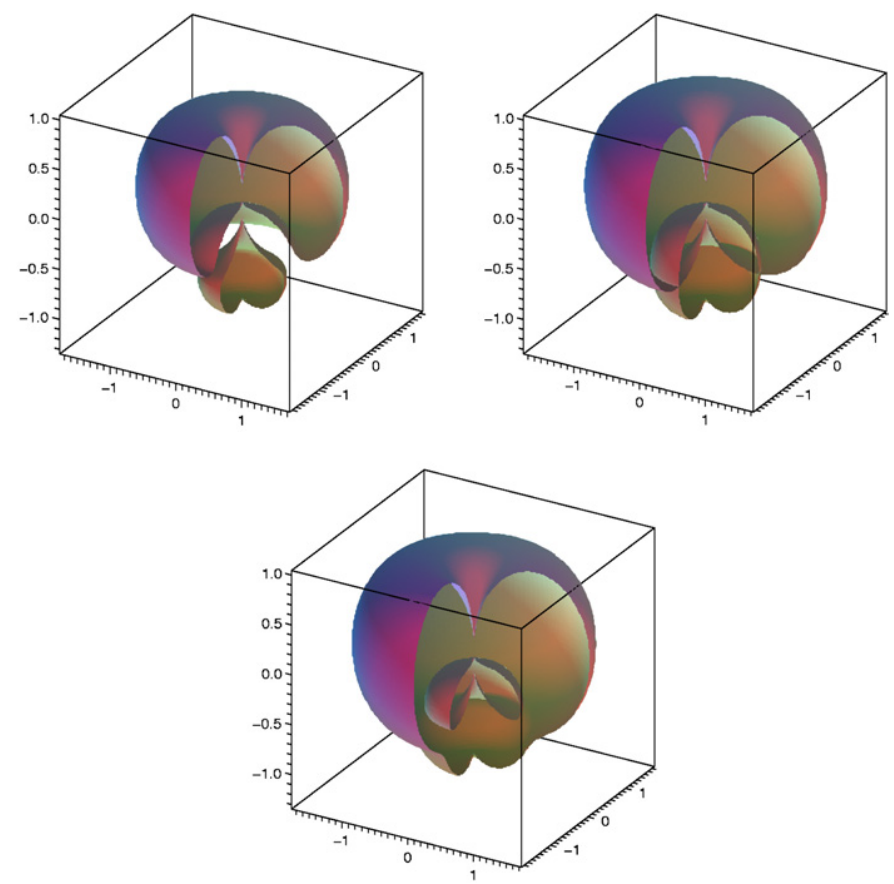

Figure 4. Coordinate representation in $\mathbb{R}^{3}$ of the first, third and fifth ergosurfaces from figure 2. The cusps where the ergosurfaces meet the symmetry axis have a geometric character and also arise in the Kerr solution [19].

We will need the following:

Proposition 5.1. Assume that $f$ and $b$ are smooth near $\vec{x}_{0}$. Then the function $b$, normalized so that $b\left(\vec{x}_{0}\right)=0$, has a zero at $\vec{x}_{0}$ of precisely the same order as $f$.

Proof. Let $k \in \mathbb{N}$ denote the order of the zero. For $k=1$ the result has already been established in section 4 , so we assume $k \geqslant 2$. We then have $f=O\left(\left|\vec{x}-\vec{x}_{0}\right|^{k}\right)$, $|D f|=O\left(\left|\vec{x}-\vec{x}_{0}\right|^{k-1}\right), \Delta f=O\left(\left|\vec{x}-\vec{x}_{0}\right|^{k-2}\right)$, and (2.5) shows that

$$
|D b|^{2}=O\left(\left|\vec{x}-\vec{x}_{0}\right|^{2 k-2}\right) .
$$

Integrating radially around $\vec{x}_{0}$ gives $b=O\left(\left|\vec{x}-\vec{x}_{0}\right|^{k}\right)$; hence the order of the zero of $b$ is greater than or equal to $k$.

To show the reverse inequality, suppose that $b=O\left(\left|\vec{x}-\vec{x}_{0}\right|^{k+1}\right)$. Inserting the Taylor expansion of $f$ into (1.1) one finds that $f_{k}$ solves the equation

$$
f_{k}\left(\frac{\partial^{2} f_{k}}{\partial \rho^{2}}+\frac{\partial^{2} f_{k}}{\partial \zeta^{2}}\right)=\left(\frac{\partial f_{k}}{\partial \rho}\right)^{2}+\left(\frac{\partial f_{k}}{\partial \zeta}\right)^{2} .
$$

On the set $\Omega_{k}:=\left\{f_{k}>0\right\}$ define $u_{k}=\ln f_{k}$. Without loss of generality, changing $f$ to $-f$ if necessary, we can assume that $\Omega_{k}$ is non-empty, with $\vec{x}_{0}$ lying in the closure of $\Omega_{k}$. On $\Omega_{k}$, equation (5.5) is simply the statement that $u_{k}$ is harmonic in the variables $(\rho, \zeta)$ :

$$
\Delta_{2} u_{k}:=\frac{\partial^{2} u_{k}}{\partial \rho^{2}}+\frac{\partial^{2} u_{k}}{\partial \zeta^{2}}=0
$$


From (5.4) we have, assuming $C \neq 0$,

$$
u_{k}=\ln C+m_{0} \ln \left(\rho-\rho_{0}\right)+\sum_{i=1}^{n} m_{i} \ln \left(\zeta-\zeta_{0}-\alpha_{i}\left(\rho-\rho_{0}\right)\right) .
$$

Inserting into (5.6) one obtains

$$
\Delta_{2} u_{k}=-m_{0} \frac{1}{\left(\rho-\rho_{o}\right)^{2}}-\sum_{i=1}^{n} m_{i}\left(1+\alpha_{i}^{2}\right) \frac{1}{\left(\zeta-\zeta_{0}-\alpha_{i}\left(\rho-\rho_{0}\right)\right)^{2}}=0 .
$$

Recalling that the $\alpha_{i}$ are distinct, this is only possible if

$$
m_{0}=0=m_{i}\left(1+\alpha_{i}^{2}\right) \quad \forall i \text {. }
$$

Reordering the $m_{i}$ if necessary, as $u_{k}$ is real valued we have proved that

$u_{k}=\ln C+m_{1} \ln \left(\left(\zeta-\zeta_{0}\right)^{2}+\left(\rho-\rho_{0}\right)^{2}\right) \quad \Longleftrightarrow \quad f_{k}=C\left(\left(\zeta-\zeta_{0}\right)^{2}+\left(\rho-\rho_{0}\right)^{2}\right)^{m_{1}}$.

Subsequently,

$$
f=C\left(\left(\zeta-\zeta_{0}\right)^{2}+\left(\rho-\rho_{0}\right)^{2}\right)^{m_{1}}+O\left(\left|\vec{x}-\vec{x}_{o}\right|^{k+1}\right)
$$

As the order of $\vec{x}_{0}$ is even, this proves proposition 5.1 for all $k$ odd.

To continue, we note the following:

Lemma 5.2. Under the hypotheses of proposition 5.1, let $\vec{x}_{0}$ be a zero of order 2. Then the quadratic form defined by the Hessian DDf $\left(\vec{x}_{0}\right)$ of $f$ has signature $(+-)$ or $(-+)$. This implies that second order zeros of $f$ are isolated.

Remark 5.3. For further use we note that the derivation of (5.8)-(5.9) only uses the truncated equations (5.13)-(5.14) below. Furthermore, the calculations here-and therefore their conclusions - remain valid when a supplementary error term $o\left(\left|\vec{x}-\vec{x}_{0}\right|^{3}\right)$ is allowed at the right-hand side of (2.5).

Proof. The result is obtained by a calculation, the simplest way proceeds as in the proof of theorem 5.6 below. Alternatively, one can use MAPLE or MATHEMATiCA, the interested reader can download the worksheets from http://th.if.uj.edu.pl/ szybka/CMS; that last calculation has been done as follows: consider the polynomials $W_{a}, a=1,2$, obtained by inserting the Taylor expansion of $f$ and $b$, with $f=D f=0$, into equations obtained by multiplying (2.5) and (2.6) with $\rho$. The requirement that those polynomials vanish up-to-and-including order 2 imposes the following alternative sets of conditions:

$$
\begin{aligned}
& \text { I. } \stackrel{\circ}{b}, 0_{2}=\stackrel{\circ}{b}_{1,1}=\stackrel{\circ}{b}, 2_{0, f_{1,1}}=0, \quad \stackrel{\circ}{f}_{2,0}=\stackrel{\circ}{f}_{0,2} \in \mathbb{R} \text {, } \\
& \text { II. } \stackrel{\circ}{f}_{2,0}=-\stackrel{\circ}{f}_{0,2}=-\grave{b}_{1,1} \in \mathbb{R}, \quad \grave{b}_{0,2}=-\stackrel{\circ}{f}_{1,1}=-\stackrel{\circ}{b}, 0_{2,0} \in \mathbb{R} \text {, }
\end{aligned}
$$

as well as a set which is related to II above by exchanging $b$ with $-b$. The first set leads to $\stackrel{\circ}{f}_{0,2}=0$ when requiring that the polynomials $W_{a}$ just defined vanish to one order higher, so that the first set cannot occur for zeros of second order. One then checks that the set II leads to Lorentzian signature of $D D f$, unless vanishing.

Clearly the Hessian of $f$ given by (5.7) does not have indefinite signature when $m_{1}=1$, proving proposition 5.1 for zeros of order 2 .

It remains to consider $m_{1} \in \mathbb{N}$ satisfying $m_{1} \geqslant 2$. Replacing $f$ by $-f$ if necessary, it follows from (5.7) that $f$ is strictly positive in a neighbourhood of $\vec{x}_{0}$, so that we can define

$$
g:=f^{1 / m_{1}} .
$$


Usual arguments (cf, e.g. [11]) show that $g$ is smooth and has a zero of order 2 at $\vec{x}_{0}$. From (2.5) one has

$$
g \Delta g-|D g|^{2}=\frac{1}{m_{1}} g^{2} \underbrace{\frac{|D b|^{2}}{f^{2}}}_{O(1)}=O\left(\left|\vec{x}-\vec{x}_{0}\right|^{4}\right) .
$$

Taylor expanding $g$ up to order $o\left(\left|\vec{x}-\vec{x}_{0}\right|^{4}\right.$ ) and inserting into (5.10) gives $C=0$ (see remark 5.3), proving proposition 5.1.

\subsection{Simple zeros}

A zero of $f$ of order $k$ will be said to be simple if all the $\alpha_{i}$ in (5.4) are real and have multiplicities one, with $m_{0} \in\{0,1\}$. We will show below that zeros of finite order of solutions of Ernst equations are simple. Somewhat to our surprise, for such zeros theorem 4.1 generalizes as follows:

Theorem 5.4. The conclusions of theorem 1.1 are valid under the supplementary condition that $f$ has only simple zeros at the $\mathscr{E}$-ergosurface $E_{f}:=\{f=0, \rho>0\}$.

Proof. As pointed out by Malgrange [11, end of section 3], simplicity implies that near $\vec{x}_{0}$ there exist smooth functions $\phi_{a}, a=1, \ldots, k$, with $\phi_{a}\left(\vec{x}_{0}\right)=0$ and with nowhere-vanishing gradient, together with a strictly positive smooth function $g$ such that we can write

$$
f=\phi_{1} \cdots \phi_{k} g \text {. }
$$

(Supposing that $m_{0}=0$, the $\phi_{a}$ have the Taylor expansion $\phi_{a}=\zeta-\zeta_{0}-\alpha_{a}\left(\rho-\rho_{0}\right)+$ $O\left(\left|\vec{x}-\vec{x}_{0}\right|^{2}\right)$; if $m_{0}=1$, then one has $\phi_{1}=\rho-\rho_{0}+O\left(\left|\vec{x}-\vec{x}_{0}\right|^{2}\right)$, with the remaining Taylor expansions of the same form as before. For $k=2$ this is a special case of Morse's theorem [6, theorem 6.9, p. 65]). Equation (5.11) shows that $E_{f}$ is, near $\vec{x}_{0}$, the union of the smooth submanifolds $\left\{\phi_{a}=0\right\}$. On each of those $D f$ is non-vanishing, except at the origin. Passing to a small neighbourhood of $\vec{x}_{0}$ if necessary, we can assume that each of the sets $\left\{\phi_{a}=0, D f \neq 0\right\}$ has precisely two components.

Consider a connected component of $\left\{\phi_{1}=0\right\}$, by section 4 equation (4.1) holds there. Suppose that the lower sign arises on this component, then the same lower sign has to arise on the remaining component of $\left\{\phi_{1}=0\right\}$, because the inversion $\vec{x}-\vec{x}_{0} \rightarrow-\vec{x}+\vec{x}_{0}$ maps each component to the accompanying one up to quadratic terms, and because $D b$ has, in the leading order of its Taylor development, the same parity as $D f$ by proposition 5.1.

We consider the function $\sigma_{\rho}$ as in (4.5), an identical argument applies to $\sigma_{\zeta}$ and to $\kappa_{\rho}, \kappa_{\zeta}$. Using a coordinate system $\left(y^{1}, y^{2}\right)$ with $\phi_{1}=y^{1}$ we have a Taylor expansion

$$
\sigma_{\rho}\left(y^{1}, y^{2}\right)=\sigma_{\rho, 0}\left(y^{2}\right)+\sigma_{\rho, 1}\left(y^{2}\right) y^{1}+\sigma_{\rho, 2}\left(y^{1}, y^{2}\right)\left(y^{1}\right)^{2} .
$$

Note that $f$ has a simple zero away from the origin on the axis $\left\{y^{1}=0\right\}$, so by the results in section 4 the functions $\sigma_{\rho, 0}$ and $\sigma_{\rho, 1}$ vanish there. By continuity they also vanish at the origin, thus $\sigma_{\rho}$ factorizes as

$$
\sigma_{\rho}=\sigma_{\rho}^{\prime} \phi_{1}^{2}
$$

for a smooth function $\sigma_{\rho}^{\prime}:=\sigma_{\rho, 2}$.

We introduce a new coordinate system $\left(z^{1}, z^{2}\right)$ in which $z^{1}=\phi_{2}$. We Taylor expand $\sigma_{\rho}^{\prime}$ as in (5.12), with the $y^{i}$ there replaced by $z^{i}$, etc. The equations,

$$
0=\left.\sigma_{\rho}\right|_{z^{1}=0, z^{2} \neq 0}=\left.\sigma_{\rho}^{\prime}\right|_{z^{1}=0, z^{2} \neq 0} \underbrace{\left.\phi_{1}^{2}\right|_{z^{1}=0, z^{2} \neq 0}}_{\neq 0},
$$


$0=\left.\mathrm{d} \sigma_{\rho}\right|_{z^{1}=0, z^{2} \neq 0}=\left.\mathrm{d} \sigma_{\rho}^{\prime}\right|_{z^{1}=0, z^{2} \neq 0} \underbrace{\left.\phi_{1}^{2}\right|_{z^{1}=0, z^{2} \neq 0}}_{\neq 0}+\left.2 \underbrace{\left.\sigma_{\rho}^{\prime}\right|_{z^{1}=0, z^{2} \neq 0}}_{=0}\left(\phi_{1} \mathrm{~d} \phi_{1}\right)\right|_{z^{1}=0, z^{2} \neq 0}$,

show that the function $\sigma_{\rho}^{\prime}$ vanishes, together with its first derivatives, away from the origin on the axis $\left\{z^{1}=0\right\}$. We conclude as before that $\sigma_{\rho}^{\prime}$ factorizes as $\sigma_{\rho}^{\prime}=\sigma_{\rho}^{\prime \prime} \phi_{2}^{2}$ for a smooth function $\sigma_{\rho}^{\prime \prime}$; hence $\sigma_{\rho}$ factorizes as

$$
\sigma_{\rho}=\sigma_{\rho}^{\prime \prime} \phi_{1}^{2} \phi_{2}^{2} .
$$

Continuing in this way, in a finite number of steps one obtains

$$
\sigma_{\rho}=\hat{\sigma}_{\rho} \phi_{1}^{2} \cdots \phi_{k}^{2}
$$

and the result easily follows.

\subsection{Zeros of finite order are simple}

Consider a zero of $f$ of order $k<\infty$, with $\rho_{0}>0$, then the leading order Taylor polynomials $f_{k}$ and $b_{k}$ solve the truncated equations

$$
\begin{aligned}
& f_{k}\left(\frac{\partial^{2} f_{k}}{\partial \rho^{2}}+\frac{\partial^{2} f_{k}}{\partial \zeta^{2}}\right)=\left(\frac{\partial f_{k}}{\partial \rho}\right)^{2}+\left(\frac{\partial f_{k}}{\partial \zeta}\right)^{2}-\left(\frac{\partial b_{k}}{\partial \rho}\right)^{2}-\left(\frac{\partial b_{k}}{\partial \zeta}\right)^{2}, \\
& f_{k}\left(\frac{\partial^{2} b_{k}}{\partial \rho^{2}}+\frac{\partial^{2} b_{k}}{\partial \zeta^{2}}\right)=2\left(\frac{\partial f_{k}}{\partial \rho} \frac{\partial b_{k}}{\partial \rho}+\frac{\partial f_{k}}{\partial \zeta} \frac{\partial b_{k}}{\partial \zeta}\right) .
\end{aligned}
$$

Let

$$
f_{k}+\mathrm{i} b_{k} \equiv \mathscr{E}_{k}=\alpha\left(z-z_{0}\right)^{k},
$$

where $\alpha \in \mathbb{C}$, with $z=\rho+\mathrm{i} \zeta$. It is straightforward, using the Cauchy-Riemann equations, to check that functions of this form satisfy (5.13)-(5.14), for all $k \in \mathbb{N}$. (In fact, both the leftand right-hand sides then vanish identically.) Those solutions have been found by inspection of the solutions found by MaPLe for $k=2$ and by Singular [7, 8] for $k=3$ and 4 . In fact, both the SINGULAR-generated solutions, as well as our remaining computer experiments using SINGULAR, played a decisive role in our solution of the problem at hand.

Let us show that

Lemma 5.5. Zeros of $f_{k}$ given by (5.15) are simple.

Proof. Indeed, the equation $f_{k}=0$ is equivalent to

$$
\alpha\left(z-z_{0}\right)^{k}=\mathrm{i} \beta,
$$

for some $\beta \in \mathbb{R}$. This is easily solved; we write $\alpha=|\alpha| \mathrm{e}^{\mathrm{i} \theta}$, and set

$$
\alpha_{\ell}=\tan \left(\frac{(2 \ell+1) \pi-2 \theta}{2 k}\right), \quad \ell=1, \ldots, k .
$$

Assuming $\alpha_{\ell} \neq \pm \infty$ for all $\ell$, we obtain $k$ distinct real lines $z_{0}+\mathbb{R}\left(1+\mathrm{i} \alpha_{\ell}\right)$ on which $\Re \mathscr{E}_{k}$ vanishes, and simplicity follows. The remaining cases are analysed similarly, and are left to the reader.

Another non-trivial, 'polarized', family of solutions of (5.13)-(5.14) is provided by $b_{k}=0, f_{k}=C\left(\left(\rho-\rho_{0}\right)^{2}+\left(\zeta-\zeta_{0}\right)^{2}\right)^{m}, m \in \mathbb{N}$. As mentioned in section 3, there exist associated static solutions of the Ernst equations. However, as already pointed out (compare remark 5.3), neither those, nor any other solutions with this $f_{k}, b_{k}$, are smooth across $E_{f}$. 
Setting $z=\rho-\rho_{0}+\mathrm{i}\left(\zeta-\zeta_{0}\right)$, the equations satisfied by $\mathscr{E}_{k}=f_{k}+\mathrm{i} b_{k}$ take the form

$$
\left(\mathscr{E}_{k}+\overline{\mathscr{E}}_{k}\right) \frac{\partial^{2} \mathscr{E}_{k}}{\partial z \partial \bar{z}}=2 \frac{\partial \mathscr{E}_{k}}{\partial z} \frac{\partial \mathscr{E}_{k}}{\partial \bar{z}}
$$

Since $\mathscr{E}_{k}$ is a polyhomogeneous polynomial in $x$ and $y$, it can be written as

$$
\mathscr{E}_{k}=\sum_{m=0}^{k} \beta_{m} z^{m} \bar{z}^{k-m}
$$

Inserting this into (5.16) we obtain

$$
\sum_{1 \leqslant m+j \leqslant 2 k-1} m \beta_{m}\left\{(2 j-k-m) \beta_{j}+(k-m) \bar{\beta}_{k-j}\right\} z^{m+j-1} \bar{z}^{2 k-m-j-1}=0 .
$$

Hence, for $1 \leqslant \ell \leqslant 2 k-1$,

$$
\sum_{m+j=\ell}\left\{(k-m) \bar{\beta}_{k-j}-(k+m-2 j) \beta_{j}\right\} m \beta_{m}=0 .
$$

Since $\ell=0$ is trivial, we obtain $2 k-2$ equations for $k+1$ numbers $\beta_{m}$, which should be rather restrictive, especially for $k \geqslant 3$. Nevertheless, as already pointed out, there exist non-trivial solutions. It is instructive to find them directly by inspection of (5.17). First, there is the obvious solution $\beta_{m}=0$ for $m \geqslant 1$, which corresponds to an anti-holomorphic $\mathscr{E}_{k}=\beta_{0} \bar{z}^{k}$. Next, one checks that a collection with $\beta_{k} \neq 0$ but $\beta_{m}=0$ for $m<k$ provides a solution, which corresponds to a holomorphic $\mathscr{E}_{k}=\beta_{k} z^{k}$. Finally, when $k=2 n$, one checks that $\beta_{n} \in \mathbb{R}$, but $\beta_{m}=0$ for $m \neq k / 2$, is a solution, which corresponds to a real $\mathscr{E}_{2 n}=\beta_{n} z^{n} \bar{z}^{n}=\beta_{n}\left(x^{2}+y^{2}\right)^{n}$.

The computer algebra program SINGULAR can be used to show that the above exhausts the list of solutions for $k$ less than or equal to eight ${ }^{11}$. This turns out to be true for all $k<\infty$ :

Theorem 5.6. These are all solutions: thus the homogeneous polynomial $\mathscr{E}_{k}$ is either holomorphic, or anti-holomorphic, or real and radial.

Proof. The case $k=1$ is a straightforward calculation, so we assume $k>1$.

If $\mathscr{E}_{k}$ is a solution, then so is its complex conjugate; this implies that if an ordered collection $\left\{\beta_{m}\right\}_{0 \leqslant m \leqslant k}$ satisfies (5.17), then so does $\left\{\bar{\beta}_{k-m}\right\}_{0 \leqslant m \leqslant k}$. Inserting this into (5.17) one obtains, again for $1 \leqslant \ell \leqslant 2 k-1$,

$$
\sum_{m+j=\ell}\left\{(k-m) \beta_{j}-(k+m-2 j) \bar{\beta}_{k-j}\right\} m \bar{\beta}_{k-m}=0 .
$$

Consider (5.17) with $\ell=1$; since $1 \leqslant m \leqslant \ell$ this enforces $m=1, j=0$, giving

$$
\left\{(k-1) \bar{\beta}_{k}-(k+1) \beta_{0}\right\} \beta_{1}=0 .
$$

Similarly (5.18) with $\ell=1$ gives

$$
\left\{(k-1) \beta_{0}-(k+1) \bar{\beta}_{k}\right\} \bar{\beta}_{k-1}=0 .
$$

Suppose, first, that $\beta_{0} \neq 0$. We will use induction arguments to establish the implication (5.21) below. So assume, for contradiction, that $\beta_{1} \neq 0$. Then $\bar{\beta}_{k}=(k+1) \beta_{0} /(k-1) \neq 0$ from (5.19), inserting into (5.20) we obtain $\beta_{k-1}=0$. But then (5.18) with $\ell=2$ gives

$$
0=\left\{(k-2) \beta_{0}-(k+2) \bar{\beta}_{k}\right\} \bar{\beta}_{k-2}=\underbrace{\frac{(k-2)(k-1)-(k+2)(k+1)}{k-1} \beta_{0}}_{\neq 0} \bar{\beta}_{k-2},
$$

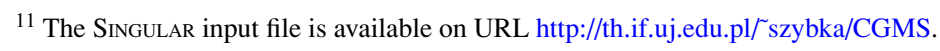


hence $\beta_{k-2}=0$. Equation (5.18) with $\ell=3$ similarly gives now $\beta_{k-3}=0$. Continuing in this way one concludes in a finite number of steps that $\beta_{1}=0$, a contradiction. It follows that $\beta_{0} \neq 0$ enforces $\beta_{1}=0$.

Assume now, again for contradiction, that $\beta_{0} \neq 0$ and $\beta_{1}=0$ but $\beta_{2} \neq 0$. Equation (5.17) with $\ell=2$ gives

$$
\left\{(k-2) \bar{\beta}_{k}-(k+2) \beta_{0}\right\} \beta_{2}=0 .
$$

If $k=2$ we obtain immediately a contradiction; otherwise $\bar{\beta}_{k}=(k+2) \beta_{0} /(k-2) \neq 0$, inserting into (5.20) we find $\beta_{k-1}=0$. But then (5.18) with $\ell=2$ gives

$$
0=\left\{(k-2) \beta_{0}-(k+2) \bar{\beta}_{k}\right\} \bar{\beta}_{k-2}=\underbrace{\frac{(k-2)^{2}-(k+2)^{2}}{k-1} \bar{\beta}_{k}}_{\neq 0} \bar{\beta}_{k-2},
$$

hence $\beta_{k-2}=0$. Continuing in this way one concludes in a finite number of steps that $\beta_{2}=0$, a contradiction. This shows that $\beta_{0} \neq 0$ and $\beta_{1}=0$ but $\beta_{2} \neq 0$ is incompatible with the equations.

It should be clear to the reader how to iterate this argument to obtain the implication

$$
\beta_{0} \neq 0 \text { implies } \beta_{m}=0 \text { for } m=1, \ldots, k \text {. }
$$

Using symmetry under complex conjugation, the hypothesis $\beta_{k} \neq 0$ leads to $\beta_{m}=0$ for $m=0, \ldots, k-1$.

It remains to analyse what happens when $\beta_{0}=\beta_{k}=0$, which we assume from now on. Suppose (for contradiction if $k>2$ ) that $\beta_{1} \neq 0$. Recalling that $k>1,(5.17)-(5.18)$ with $\ell=2$ give

$$
\left(\bar{\beta}_{k-1}-\beta_{1}\right) \beta_{1}=0=\left(\beta_{1}-\bar{\beta}_{k-1}\right) \bar{\beta}_{k-1} .
$$

If $k=2$ we obtain $\beta_{1} \in \mathbb{R}$, and we are done.

Otherwise $\beta_{k-1}=\bar{\beta}_{1} \neq 0$ and (5.18) with $\ell=3$ gives

$$
\left\{(k-1) \beta_{2}-(k+1) \bar{\beta}_{k-2}\right\} \beta_{1}=0 .
$$

When $k=3$ this gives a contradiction, and the result is established for this value of $k$.

For $k \geqslant 4$ the proof will be finished by more induction arguments, as follows: suppose, to start with, that $\beta_{k}=0$ and that there exist $k_{0}, k_{1} \in \mathbb{N}, 1 \leqslant k_{1} \leqslant k_{0} \leqslant k / 2$, such that $\beta_{m}=0$ for $0 \leqslant m \leqslant k_{0}-1$ and for $k-k_{1}<m \leqslant k$ but $\beta_{k_{0}} \neq 0$. (The case $k_{1}>k_{0}$ can be reduced to this one by replacing $\mathscr{E}_{k}$ with its complex conjugate.) With these hypotheses (5.17) can be rewritten as

$$
\sum_{k_{0} \leqslant m \leqslant \min \left(k-k_{1}, \ell-k_{1}\right)}\left\{(k-m) \bar{\beta}_{k-(\ell-m)}-(k+3 m-2 \ell) \beta_{\ell-m}\right\} m \beta_{m}=0 .
$$

Equation (5.22) with $\ell=k_{0}+k_{1} \leqslant k$ gives

$$
\left(k-k_{0}\right) \bar{\beta}_{k-k_{1}}=\left(k+k_{0}-2 k_{1}\right) \beta_{k_{1}}
$$

which equals zero unless $k_{1}=k_{0}$. It follows that we can without loss of generality assume that $k_{1}=k_{0}$ and

$$
\beta_{k-k_{0}}=\bar{\beta}_{k_{0}} .
$$

We can now rewrite $(5.18)$ as

$\sum_{k_{0} \leqslant m \leqslant \min \left(k-k_{0}, \ell-k_{0}\right)}\left\{(k-m) \beta_{\ell-m}-(k+3 m-2 \ell) \bar{\beta}_{k-(\ell-m)}\right\} m \bar{\beta}_{k-m}=0$. 
Suppose that $k=2 k_{0}$; then (5.23) leads immediately to the restriction $\beta_{k_{0}} \in \mathbb{R}$, giving a real radial solution, as desired. Otherwise, choosing $\ell=2 k_{0}+1$ in (5.23) one obtains

$$
\left(k-k_{0}\right) k_{0} \beta_{k_{0}+1}=\left[\left(k-k_{0}\right) k_{0}+2\right] \bar{\beta}_{k-k_{0}-1} .
$$

Equation (5.22) with $\ell=2 k_{0}+1$ gives

$$
\left(k-k_{0}\right) k_{0} \bar{\beta}_{k-k_{0}-1}=\left[\left(k-k_{0}\right) k_{0}+2\right] \beta_{k_{0}+1} .
$$

It follows that

$$
\beta_{k_{0}+1}=\beta_{k-k_{0}-1}=0 \text {. }
$$

Our aim now is to show (5.24) below, by a last induction. So, suppose there exists $k_{2} \in \mathbb{N}$ satisfying $k_{0}<k_{2}<k-k_{0}$ such that $\beta_{m}=0$ for $k_{0}<m<k_{2}$ and for $k-k_{2}<m<k-k_{0}$; we have shown that this is true with $k_{2}=k_{0}+2$. Equation (5.23) with $\ell=k_{0}+k_{2}$ gives

$$
\left(k-k_{0}\right) k_{0} \beta_{k_{2}}=\left[\left(k-k_{0}\right) k_{0}+2\left(k_{2}-k_{0}\right)^{2}\right] \bar{\beta}_{k-k_{2}} .
$$

But from (5.22) again with $\ell=k_{0}+k_{2}$ one obtains

$$
\left(k-k_{0}\right) k_{0} \bar{\beta}_{k-k_{2}}=\left[\left(k-k_{0}\right) k_{0}+2\left(k_{2}-k_{0}\right)^{2}\right] \beta_{k_{2}} .
$$

This allows us to conclude that

$\beta_{m}=0 \quad$ except if $m=k_{0} \quad$ or if $m=k-k_{0}$, with $\quad \beta_{k-k_{0}}=\bar{\beta}_{k_{0}}$.

Equation (5.23) with $\ell=k$ gives now $\beta_{k_{0}}=0$ (recall that we have assumed $k \neq 2 k_{0}$ ), a contradiction, and the theorem is proved.

We can now pass to the

Proof of theorem 1.1. Theorem 5.6 gives the list of all possible $\mathscr{E}_{k}$. The real ones do not lead to smooth $f$ by proposition 5.1. The holomorphic ones lead to simple zeros by lemma 5.5; the same is true for the anti-holomorphic ones, because the condition of simplicity is preserved by complex conjugation of $\mathscr{E}$. The result follows now from theorem 5.4.

\section{Acknowledgments}

We wish to thank G Alekseev, M Ansorg, R Beig, M-F Bidaut-Véron, M Brickenstein, S Janeczko, J Kijowski, G Neugebauer, D Petroff and L Véron for useful comments or discussions. We acknowledge hospitality and financial support from the Newton Institute, Cambridge (PTC, RM, SSz), as well as the AEI, Golm (PTC) during work on this paper. PTC was partially supported by a Polish Research Committee grant 2 P03B 073 24. RM was partially supported by the Deutsche Forschungsgemeinschaft SFB/TR7-B1. SSz was partially supported by a Polish Research Committee grant 1 P03B 01229.

\section{References}

[1] Ansorg M 2001 Differentially rotating disks of dust: arbitrary rotation law Gen. Rel. Grav. 33 309-38 (Preprint gr-qc/0006045)

[2] Ansorg M and Petroff D 2005 Black holes surrounded by uniformly rotating rings Phys. Rev. D 72024019 (Preprint gr-qc/0505060)

[3] Carter B 1973 Black hole equilibrium states Black Holes (Proc. Les Houches Summer School) ed C de Witt and B de Witt (New York: Gordon and Breach)

[4] Chruściel P T, Meinel R and Szybka S 2005 The Ernst equation and ergosurfaces (Newton Institute) Preprint NI05081-GMR, http://www.newton.cam.ac.uk/preprints/NI05081.pdf

[5] Ernst F J 1968 New formulation of the axially symmetric gravitational field problem Phys. Rev. 167 $1175-8$ 
[6] Golubitsky M and Guillemin V 1973 Stable Mappings and Their Singularities (Graduate Texts in Mathematics vol 14) (New York: Springer)

[7] Greuel G-M and Pfister G 2002 A Singular Introduction to Commutative Algebra with contributions by ed O Bachmann, C Lossen and H Schönemann (Berlin: Springer) (MR MR1930604 (2003k:13001))

[8] Greuel G-M, Pfister G and Schönemann H 2002 Singular, a computer algebra system for polynomial computations http://www.singular.uni-kl.de

[9] Kramer D and Neugebauer G 1979-1980 The superposition of two Kerr solutions Phys. Lett. A 75 259-61 (MR MR594394 (81m:83014))

[10] Lewandowski J and Pawłowski T 2003 Extremal isolated horizons: a local uniqueness theorem Class. Quantum Grav. 20 587-606 (Preprint gr-qc/0208032)

[11] Malgrange B 2003 Idéaux de fonctions différentiables et division des distributions Distributions Ed. Éc. Polytech. Palaiseau pp 1-21 http://www.math.polytechnique.fr/xups/vol03.html. MR MR2065138

[12] Manko V S and Ruiz E 1998 Extended multi-soliton solutions of the Einstein field equations Class. Quantum Grav. 15 2007-16 (MR MR1633190 (99m:83045))

[13] Müller zum Hagen H 1970 On the analyticity of stationary vacuum solutions of Einstein's equation Proc. Camb. Phil. Soc. 68 199-201 (MR 41 \#5017)

[14] Neugebauer G 1980 A general integral of the axially symmetric stationary Einstein equations J. Phys. A: Math. Gen. 13 L19-21 (MR MR558632 (80k:83024))

[15] Neugebauer G, Kleinwächter A and Meinel R 1996 Relativistically rotating dust Helv. Phys. Acta $77472-89$

[16] Neugebauer G and Meinel R 1995 General relativistic gravitational field of a rigidly rotating disk of dust: solution in terms of ultraelliptic functions Phys. Rev. Lett. 753046

[17] Neugebauer G and Meinel R 2003 Progress in relativistic gravitational theory using the inverse scattering method J. Math. Phys. 44 3407-29 (Preprint gr-qc/0304086)

[18] Oohara K and Sato H 1981 Structure of superposed two Kerr metrics Prog. Theor. Phys. 65 1891-900 (MR MR626966 (82m:83009))

[19] Pelavas N, Neary N and Lake K 2001 Properties of the instantaneous ergo surface of a Kerr black hole Class. Quantum Grav. 18 1319-32 (Preprint gr-qc/0012052)

[20] Rueda J A, Manko V S, Ruiz E and Sanabria-Gomez J D 2005 The double-Kerr equilibrium configurations involving one extreme object Class. Quantum Grav. 22 4887-94 (Preprint gr-qc/0508101)

[21] Weinstein G 1990 On rotating black-holes in equilibrium in general relativity Commun. Pure Appl. Math. 43 903-48

[22] Yamazaki M 1983 Stationary line of N Kerr masses kept apart by gravitational spin-spin interaction Phys. Rev. Lett. 50 1027-30 (MR MR700070 (84e:83021)) 